patients $(55.8 \%)$ had presented anemia of grade $1-2$ and 18 patients (34.6\%) had experienced anemia of grade $3-4$, which seemed higher than the previous reports. Five patients received blood transfusions. As for non-hematological toxicity, nausea (25 patients, $48.1 \%$ ), fatigue (20 patients, 38.4\%) and blood creatinine increased (27 patients, $51.9 \%$ ) were observed frequently (any grade). The incidence of blood creatinine increased also seemed higher than the previous studies. One patient discontinued olaparib due to prolonged renal dysfunction. In total, 22 patients (42.3\%) required dose interruption, and 11 patients $(21.2 \%)$ required dose reduction. No patient had suffered from acute myeloid leukemia or myelodysplastic syndromes.

Conclusion: Olaparib maintenance monotherapy was tolerable also for Japanese population, although the toxicity profile might be a bit different from the previous reports (SOLO2, Study 19).

Poster (022)

Epithelial Ovarian Cancer including Borderline Tumor

https://doi.org/10.3802/jgo.2021.32.S1.022

\section{TP53 sequence analysis revealed distinct characteristics of p53 signatures in RRSO samples}

Kenta Masuda," Tomoko Akahane, Yusuke Kobayashi, Tatsuyuki Chiyoda, Kouji Banno, Daisuke Aoki

Keio University School of Medicine, Tokyo, Japan (ma-su-ken.a2@keio.jp)

Objective: Risk-reducing salpingo-oophorectomy (RRSO) is recommended for women with $B R C A 1 / 2$ pathogenic variants upon completion of childbearing. Precursor lesions could be identified in the fallopian tubes of RRSO samples. Serous tubal intraepithelial carcinoma (STIC) is considered a precursor of high-grade serous carcinoma (HGSC), however, the significance of the p53 signature remains unclear. In this study, we analyzed the potency of the p53 signature as a precancerous lesion. Methods: We analyzed the clinicopathological findings and conducted DNA sequencing for TP53 variants of $\mathrm{p} 53$ signatures isolated using laser capture microdissection in 13 patients with $B R C A 1 / 2$ pathogenic variants who underwent RRSO and 17 control patients with the benign gynecologic disease.

Results: p53 signatures were most frequently observed in fimbriae of fallopian tubes from RRSO samples. Between the RRSO group and the control group, there was no significant difference in the percentage of $\mathrm{p} 53$ signatures in fimbriae $(\mathrm{p}=0.17)$, whereas the percentage of pathogenic variants in TP53 variants identified in $\mathrm{p} 53$ signature lesions were significantly higher in the RRSO group than the control $(\mathrm{p}<0.001)$. Conclusion: The characteristics of the $\mathrm{p} 53$ signature may be different between specimens obtained during RRSO and those from controls. The sequence analysis for TP53 revealed distinct characteristics of $\mathrm{p} 53$ signatures as precancerous lesions.

Poster (023)

Non-epithelial Ovarian Cancer

https://doi.org/10.3802/jgo.2021.32.S1.023

\section{Tumor recurrence and fertility in young women with malignant ovarian germ cell tumors who received fertility-sparing surgery}

\section{Seok Mo Kim, " U Chul Ju, Woo Dae Kang}

Department of Obstetrics and Gynecology, Chonnam National University Medical School, Gwangju, Korea (ddiamo94@gmail.com)

Objective: We evaluated the tumor recurrence and fertility outcomes in young women with malignant ovarian germ cell tumors (MOGCTs) who received fertility-sparing surgery (FSS). Methods: We reviewed the medical records of women aged $\leq 40$ years with MOGCTs who received FSS between July 2002 and December 2018, retrospectively. FSS was defined as the preservation of the uterus and at least one adnexa.

Results: Forty-four women were included in the study. The median age of the patients was 22 years (range, 7-39 years). Twenty-nine patients $(65.9 \%)$ received postoperative chemotherapy with bleomycin, etoposide, and cisplatin. During a median follow-up period of 62 months (range, 7-185), 4 patients $(9.1 \%)$ had a recurrence. Of these, 2 patients with dysgerminoma had recurrences at para-aortic lymph nodes and 2 patients with immature teratomas had recurrences at the remaining ovary. Of a total of 44 patients, $37(88.1 \%)$ had regular menstruations. Of 14 women desiring a pregnancy, 12 achieved the term delivery of 12 singleton pregnancies. The pregnancy and live birth rates were $85.7 \%$ and $100 \%$, respectively.

Conclusion: FSS with or without adjuvant chemotherapy is an appropriate option for young women with MOGCTs who wish to preserve their fertility.

Poster (024)

Non-Epithelial Ovarian Cancer

https://doi.org/10.3802/jgo.2021.32.S1.024

\section{Giant ovarian fibrosarcoma-a rare case report}

Pratibha Kumari," Satya Kumari, Sangeeta Pankaj

IGIMS, Patna, India (Pratibhabseb@gmail.com)

Objective: Ovarian fibrosarcoma is very rare malignant neoplasm, accounts for less than $1 \%$ of all ovarian 
malignancies, usually seen in peri- or post-menopausal women. Criteria for diagnosis of fibrosarcoma include mitotic count, the most important feature for distinguishing between benign and malignant lesions. Only few cases have been reported due to its low incidence and poor prognosis. These tumors are easily misdiagnosed so sometimes diagnosis is primarily based on histopathological and immunohistochemistry (IHC) report as in our case report. We report a rare case of ovarian fibrosarcoma. Methods: A 40 year, P5L5, woman visited to gynecological oncology department of IGIMS, Patna, with complain of abdominal distention for 6 months. Her menstrual history was regular with average flow. On abdominal examination-a lump of 32 week size with mobility found. On vaginal examinationcervix was healthy, uterine size could not be assessed due to such huge mass. Her tumor markers showed raised cancer antigen 125 level with value of 152.7. Other tumor markers were within normal limit. Computed tomography abdomen showed $22 \times 19 \times 16 \mathrm{~cm}$ mass in left adenexa with multiple septae and few cystic areas.

Results: Primary debulking surgery done. Final pathological diagnosis was fibrosarcoma of left ovary. IHC showed ki-67-35\%. Conclusion: Primary ovarian fibrosarcomas is a rare neoplasm of ovary but it must be considered as differential diagnosis of unilateral huge solid ovarian mass in all age group. ki-67 along with mitosis, greater than 4 mitotic areas/HPF, has recently become an important indicator for diagnosis of ovarian fibrosarcoma. Preoperative diagnosis is often difficult. Gynaeoncologist has to wait for histopathological and IHC report for final diagnosis.

Poster (025)

Rare Tumors \& Metastatic Tumors

https://doi.org/10.3802/jgo.2021.32.S1.025

\section{Mature cystic teratoma of the ovary with keratinizing squamous cell carcinoma}

\section{Renardy Reza Razali, ' Kartiwa Hadi Nurianto,} Primariadewi Rustamadji

Faculty of Medicine, Indonesia University, Jakarta, Indonesia (renardireza@gmail.com)

Objective: Learning about clinical manifestation and diagnosis of mature teratoma cases with malignant transformation. Methods: A 41-year-old woman, P3A0, was referred from primary hospital because of post-operative pathological examination revealed a malignancy. Earlier, she underwent subtotal abdominal hysterectomy and bilateral salpingooophorectomy at primary hospital for indication abdominal enlargement and dysmenorrhea. After procedure, post- operative pathological result revealed; keratinized squamous cell carcinoma, poorly differentiated originating from mature teratoma of the right ovary. Tumor cell infiltration was seen in the tissue around the right tube.

Results: Histological according to keratinized squamous cell carcinoma, poorly differentiated originating from mature teratoma of the right ovary.

Conclusion: Malignant transformation of benign ovarian tumors, including mature teratoma, is very rare. In addition, the clinical findings and pre-operative examinations did not help a lot to establish the diagnosis. Often the diagnosis was discovered unexpectedly in post-operative specimens such as happened in this case.

Poster (026)

Epithelial Ovarian Cancer including Borderline Tumor https://doi.org/10.3802/jgo.2021.32.S1.026

\section{Preoperative evaluation for prediction of suboptimal primary cytoreductive surgery in advanced-stage ovarian cancer}

Ruai Kittikhun, "Witsarut Apikijmeta, Suttida Intharaburan

Phramongkutklao Hospital, Bangkok, Thailand (ruai.kittikhun@gmail.com)

Objective: To identify the preoperative predicting factors of suboptimal primary cytoreductive surgery (PCS) in advanced stage ovarian cancer patients and to determine the suboptimal cytoreduction rate.

Methods: Retrospective cross-sectional study was conducted between 1 January 2014 to 31 December 2019. Ovarian cancer International Federation of Gynecology and Obstetrics (FIGO) stage III-IV patients who underwent PCS in Phramongkutklao Hospital were reviewed. Data collection included age, performance status, serum cancer-antigen 125 (CA125) and radiological criteria from computed tomographic (CT) scan of whole abdomen with intravenous and oral contrast provided within 30 days to PCS. Univariate and multivariate logistic regression analysis were employed to access the predicting factors of suboptimal PCS. The suboptimal cytoreduction rate was presented in percentage.

Results: Eighty patients with advanced ovarian cancer who had PCS by gynecologic oncologist in Phramongkutklao Hospital were enrolled. The majority of ovarian cancer FIGO stage in this population was IIIC (60\%). The suboptimal surgery rate in the study was $62.5 \%$. According to univariate analysis, Eastern Cooperative Oncology Group performance score $=1-3$ $(p=0.017)$, serum CA125 level of 500 units $/ \mathrm{mL}$ or more $(p=0.02)$, CT features of retroperitoneal lymph nodes above renal hilum 